\title{
Chemcomm
}

Chemical Communications

rsc.li/chemcomm

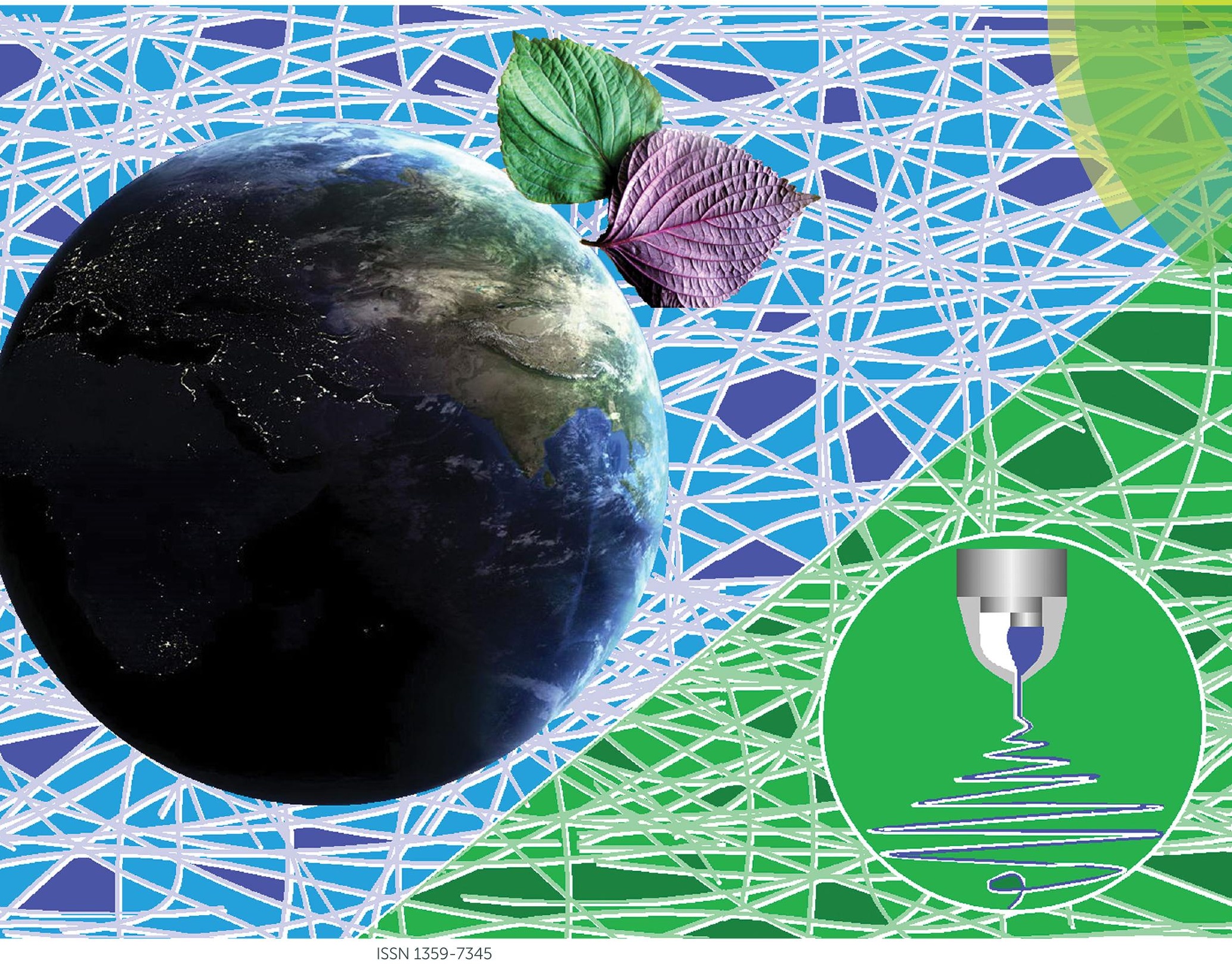




\title{
ChemComm
}

Check for updates

Cite this: Chem. Commun., 2017 53,4542

Received 3rd March 2017

Accepted 31st March 2017

DOI: $10.1039 / \mathrm{c7cc01661a}$

rsc.li/chemcomm

\section{High-quality Janus nanofibers prepared using three-fluid electrospinning $\dagger$}

\author{
Deng-Guang Yu, ${ }^{\text {a }}$ Jiao-Jiao Li, ${ }^{a}$ Man Zhang ${ }^{a}$ and Gareth R. Williams (D) *b
}

\begin{abstract}
A structured spinneret comprising two acentric needles nested into a third metal capillary was developed to conduct three-fluid electrospinning processes. With an exterior solvent surrounding two core fluids arranged side-by-side, high quality polyvinylpyrrolidone/ shellac Janus nanofibers could be prepared, which was not possible using a standard side-by-side spinneret without this innovation.
\end{abstract}

The relationship between a material's structure and its functional performance, and the ability to tune these in a systematic manner, are of vital importance for the intelligent design of an effective product. This is equally true on the macroscale and the nanoscale. A range of different nanoscale structures have been reported, but the core-shell and Janus structures are the most fundamental of these. ${ }^{1,2}$ The former provides an inner-outer relationship between two compartments, and the latter a sideby-side arrangement. Both frequently appear in nature and are similarly useful starting points for creating man-made materials to provide multiple functions, or to exploit the synergetic action of two components. ${ }^{2}$ Other, more complex, nanoscale structures can be viewed as combinations and derivatives of these two basic architectures. Unlike core-shell structures, almost all the Janus nanomaterials reported to date have been synthesized by bottom-up routes, such as molecular self-assembly. ${ }^{3-5}$

Electrospinning is a facile top-down method for creating nanofibers, and the past two decades have seen the publication of many studies using this technique to generate products for a wide variety of potential applications. ${ }^{6-8}$ The technique essentially involves making a solution of a polymer (often with a functional component) in a volatile solvent, and ejecting this from a syringe fitted with a metal needle (the spinneret). A high potential difference is applied between the spinneret and a

\footnotetext{
${ }^{a}$ School of Materials Science \& Engineering, University of Shanghai for Science and Technology, Shanghai 200093, P. R. China. E-mail: ydg017@usst.edu.cn

${ }^{b}$ UCL School of Pharmacy, University College London, 29-39 Brunswick Square, London WC1N 1AX, UK. E-mail: g.williams@ucl.ac.uk

$\dagger$ Electronic supplementary information (ESI) available: Detailed experimental procedures, characterization of materials, and additional results concerning the spinning of PVP and different shellac solutions. See DOI: 10.1039/c7cc01661a
}

grounded collector, and the electrical energy causes rapid evaporation of the solvent and yields one-dimensional polymerbased fibers. ${ }^{9,10}$ Most reports have focused on the advantageous properties of the non-woven fiber mats which result from electrospinning, such as their very high large surface area and high porosity. ${ }^{11-14}$ However, the most attractive feature of electrospinning is its ability to generate nanoscale structures from a macroscale spinneret, thereby allowing the development of complex nanostructures in one step.

To date, although there are many publications detailing monolithic fibers from single-fluid spinning, reports concerning complex (e.g. core-shell, Janus) electrospun nanostructures are rather limited, and most concern core-shell nanofibers. Although electrospun three- and four-layer core-shell materials have been reported recently, ${ }^{15-18}$ investigations into electrospun Janus products are rare, with only ten or so publications during the past 20 years. ${ }^{19-30}$ This situation is related to the great difficulty that arises in producing Janus fibers using a one-step "top-down" process. ${ }^{31,32}$

The key issue underpinning this difficulty lies in the need to manipulate working fluids with very different properties, and ensure that these are drawn synchronously from the spinneret without separation under the electrical field. A carefully considered spinneret design is crucial: ${ }^{33}$ the spinneret must both provide a template for generating the desired nanoscale structure, and also control the behavior of the working fluids under the electrical field.

Three different spinnerets were developed for implementing side-by-side electrospinning in this work. These are termed parallel (spinneret I, which has been reported several times in the literature ${ }^{19-30}$ ), acentric (II), and structured (III), and are respectively shown in Fig. 1a, d and g. Three fluids were used. The first (spinnable) fluid was a transparent $8 \%(\mathrm{w} / \mathrm{v})$ polyvinylpyrrolidone (PVP) K60 solution in ethanol, the second (spinnable) a magenta $80 \%(\mathrm{w} / \mathrm{v})$ shellac solution in ethanol, and the third (unspinnable) pure ethanol. PVP and shellac were chosen because, based on our previous experience, we know them to have very different behavior under an electric field 


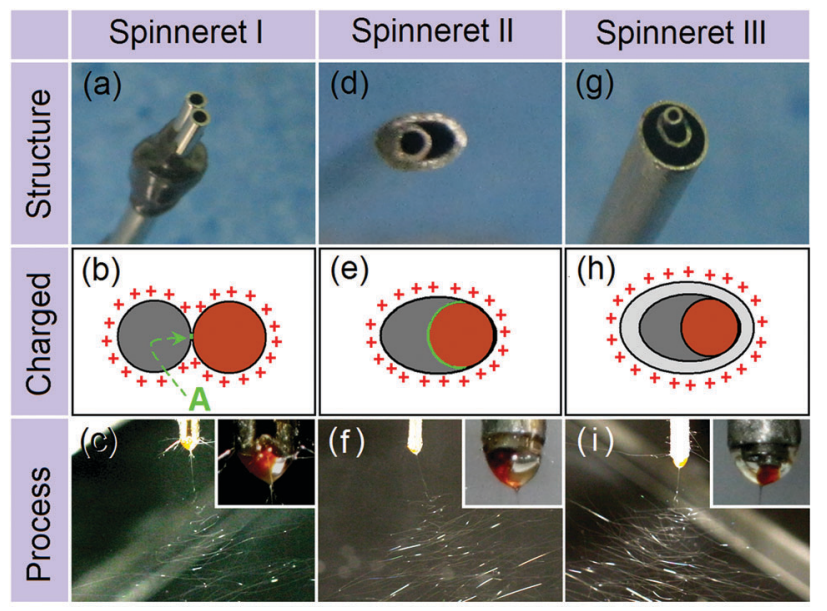

Fig. 1 Side-by-side electrospinning using different spinnerets. (a, $d$ and g) are photographs of the parallel, acentric, and structured spinnerets, respectively; ( $b$, e and $h$ ) are schematics of the charged area and fluid contact interfaces; and ( $c, f$ and i) are typical photographs of the spinning processes (insets: Taylor cones)

(for instance, the polymer concentration required to make a spinnable solution is some 5 times greater for shellac than for PVP), and thus they make excellent model polymers. They also have very different dissolution properties in water, meaning they have great potential in dual-compartment drug delivery systems.

Fig. $1 \mathrm{~b}$ and $\mathrm{c}$ illustrate the electrical charges on the working fluids and a photograph of a typical experiment using the two spinnable fluids and spinneret $\mathrm{I}$. This results in a small contact area between the two working fluids (marked "A" in Fig. 1b), as well as a large and irregular charged surface. Fig. 1e and $f$ show a schematic of the charges on the working fluids and a photograph for the spinning process using spinneret II. Compared to spinneret I, the acentric spinneret results in a much larger contact area between the working fluids, as well as a smaller and more continuous charged surface.

Recent work has shown that the introduction of an additional, pure solvent, fluid around the spinnable working fluids can be beneficial in increasing the quality of the nanofibers produced, and in preventing clogging at the spinneret. ${ }^{34-36}$ Based on these findings, a third structured spinneret based on II, but incorporating an additional shell compartment was prepared (spinneret III). The charges on the working fluids and a photograph of the resultant spinning process (with the two spinnable fluids in the core compartments, and pure ethanol in the shell) are given in Fig. $1 \mathrm{~h}$ and i, respectively.

Although both the Taylor cones (insets of Fig. 1c, f, and i) and the bending and whipping zones using all three spinnerets appear perfect, SEM images of the fibers produced tell a different story. There are clear differences in the fibers' structural integrity, size, and size uniformity. The product from spinneret I (Fig. 2a) contains two populations of fibers, a result of the two working fluids separating after exiting the spinneret. One population (likely to be from the PVP K60 solution given its low $8 \% \mathrm{w} / \mathrm{v}$ concentration) had much narrower diameters than the other (comprising shellac). These findings suggest a failure

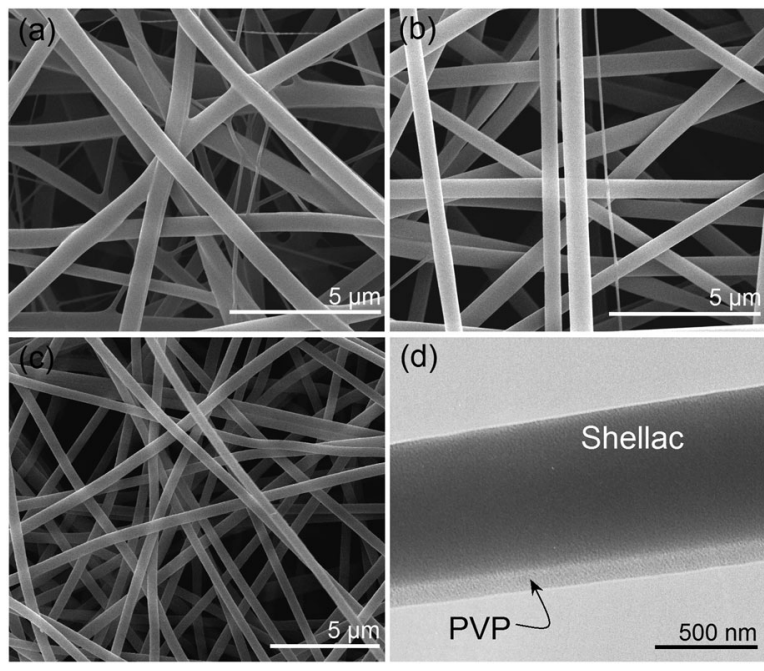

Fig. 2 The morphologies of the fibers prepared. (a-c) SEM images of the fibers from spinnerets I, II and III, respectively; (d) a TEM image of the Janus structures from spinneret III. The average fiber diameters are $1130 \pm$ $170 \mathrm{~nm}$ (spinneret I), $940 \pm 120 \mathrm{~nm}$ (II) and $710 \pm 50 \mathrm{~nm}$ (III).

in preparing high-quality Janus fibers with structural integrity. The reasons underlying the fluid separation are as follows: (1) the small contact area of the two working fluids; (2) both fluids have the same electrical charge and thus repel; and (3) the two fluids solidify at different rates.

The fibers from the acentric spinneret show improved quality, with fewer detached PVP fibers and a narrow distribution of fiber diameters (Fig. 2b). However, some polymer separation can still be observed. Although the two fluids had a continuous charged surface under spinneret II, they still retain different properties. Thus, their behaviors under the electrical field and their solidification rates are non-identical. The shellac concentration is $80 \% \mathrm{w} / \mathrm{v}$ and that of PVP only $8 \% \mathrm{w} / \mathrm{v}$, so there is much less solvent to remove from the shellac working fluid, and this will solidify first. The PVP jet could still be drawn after the shellac solution had solidified, which results in the detachment of the PVP fluid from the shellac side.

SEM and TEM images of the fibers from spinneret III are depicted in Fig. 2c and d, respectively. Clearly, these fibers have perfect Janus structures, with full structural integrity, narrow diameters and a uniform size distribution. During spinning, the surrounding ethanol solvent could act as a protective shell to the side-by-side shellac/PVP fluids. Given that charges induced under an electrical field are always distributed on the exterior surface of the working fluids, the ethanol shell can completely eliminate any electrostatic repulsion between the two core fluids (Fig. 1h). It can also ensure that the two core fluids are drawn and dried synchronously.

Given the large body of literature reporting core/shell fibers from coaxial spinning with a concentric spinneret, ${ }^{37,38}$ it might be expected that a spinneret consisting of two parallel metal capillaries could be used for generating Janus fibers. However, the parallel spinneret I cannot easily ensure the production of an integrated Janus structure. The suggested mechanism 
(a)

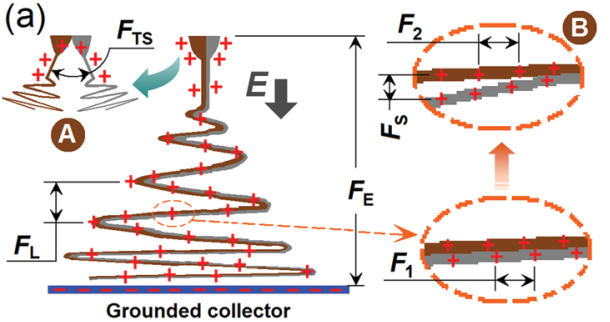

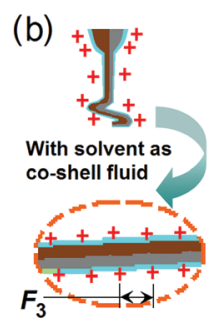

Fig. 3 The formation mechanisms of fibers prepared using spinnerets (a) I and (b) III.

underlying this is shown in Fig. 3a. The identical charges on the two fluids and small contact area between them might allow the two fluids to become detached from each other early in the spinning process (either in the Taylor cone or the straight jet) because of the repulsive force $F_{\mathrm{TS}}$ between them ("A" in Fig. $3 \mathrm{a}$ ). In the data reported thus far, this phenomenon was not observed because the two spinnable fluids were compatible and sticky enough to bind together. When the shellac concentration was reduced, such a separation phenomenon was frequently observed for spinneret I, but not for spinneret II (see ESI $\dagger$ ). The separation of the two sides will be further encouraged when the two fluids enter into the unstable region. Here, they are subjected to a series of forces, which include the Coulombic repulsion forces $\left(F_{1}\right.$ and $F_{2}$ ) from the surface charges on the fluid jets, attractive forces between the two electrodes $\left(F_{\mathrm{E}}\right)$, repulsion forces between adjacent bending coils $\left(F_{\mathrm{L}}\right)$, repulsion forces between the two sides of the Janus fluid jets $\left(F_{\mathrm{S}}\right)$, and gravitational forces. The fact that $F_{1}$ and $F_{2}$ will not be identical, and the action of $F_{\mathrm{S}}$, will directly result in the separation of the two fluids ("B" in Fig. 3a), and the other forces might exacerbate this.

When the acentric spinneret II was used, the Janus fibers' quality can be improved, since this offers a number of advantages over spinneret I. First, a closed-loop charged surface is ensured because of the nozzle shape, and there is a large contact area between the two working fluids. These factors facilitate the formation of a compound side-by-side Taylor cone. This first step of the electrospinning process is very important for the later emission of side-by-side straight fluid jet, and synchronous bending and whipping of the two fluids. Based on this idea, previous publications report the creation of integrated Janus nanofibers though intelligent spinneret design, including those with varied port-angles ${ }^{31}$ or a Teflon coating. ${ }^{32}$ It is expected that the acentric spinneret II could be advantageous over these, with less energy loss to the environment (for the reasons described above). ${ }^{39,40}$ As a result, more electrostatic energy can be transferred to the working fluids to draw and dry them. However, the acentric spinneret II, just as its varied port-angle and Teflon-coated predecessors, cannot exert any influence on the bending and whipping processes, which are believed to be the most important parts of the drying process in terms of narrowing the fluid jets and converting them to fibers. ${ }^{41}$

When a solvent surrounding both fluids was added through spinneret III, a common Coulombic repulsion force $F_{3}$ is

exerted on the shell fluid during the bending and whipping of the multiple fluids (Fig. 3b), and these forces should be transmitted from the outer surface to the inner parts. Thus, the two core fluids are drawn and dry synchronously, at least in the early stages of the unstable region.

In summary, high-quality Janus nanofibers can be easily fabricated using a three-fluid electrospinning process and a structured spinneret. This experimental design can effectively manipulate the behavior of the fluids from the Taylor cone to the bending and whipping region under the electrical field. The production of a Janus structure can be ensured because the spinneret: (1) gives a relatively high contact area between the two polymer solutions; (2) reduces repulsive forces between the two fluids in the early stages of the electrospinning process; and (3) facilities the two fluids moving and drying simultaneously. The exploration of electrospinning for the creation of complicated nanostructures is still at its infancy, and thus we believe these findings have great potential in the development of advanced materials. The two concepts reported here can pave the way to develop a broad series of new multiple-fluid electrospinning processes.

This work was supported by the Natural Science Foundation of China (NSFC; No. 51373101) and the NSFC/UK Royal Society International Exchanges Scheme (No. 51411130128/IE131748).

\section{Notes and references}

1 R. G. Chaudhuri and S. Paria, Chem. Rev., 2012, 112, 2373.

2 A. Walther and A. H. E. Müller, Chem. Rev., 2013, 113, 5194.

3 S. Bhaskar and J. Lahann, J. Am. Chem. Soc., 2009, 131, 6650.

4 H. Chen, Y. Zhao, Y. Song and L. Jiang, J. Am. Chem. Soc., 2008, 130, 7800.

5 J. D. Starr and J. S. A. Andrew, J. Mater. Chem. C, 2013, 1, 2529.

6 J. Hu, S. Zhou, Y. Sun, X. Fang and L. Wu, Chem. Soc. Rev., 2012, 41, 4356.

7 Janus Particle Synthesis, Self-assembly and Applications, ed. S. Jiang and S. Granick, RSC, Cambridge, UK, 2012.

8 A. H. Gröschel and A. H. Müller, Nanoscale, 2015, 7, 11841.

9 D. Li and Y. Xia, Adv. Mater., 2004, 16, 1151.

10 S. Agarwal, A. Greiner and J. H. Wendorff, Prog. Polym. Sci., 2013, 38, 963.

11 S. Ramakrishna, K. Fujihara, W. E. Teo, T. Yong, Z. Ma and R. Ramaseshan, Mater. Today, 2006, 9, 40.

12 B. Sun, Y. Z. Long, H. D. Zhang, M. M. Li, J. L. Duvail, X. Y. Jiang and H. L. Yin, Prog. Polym. Sci., 2014, 39, 862.

13 S. Peng, G. Jin, L. Li, K. Li, M. Srinivasan, S. Ramakrishna and J. Chen, Chem. Soc. Rev., 2016, 45, 1225.

14 K. Molnar and Z. K. Nagy, Eur. Polym. J., 2016, 74, 279.

15 D. Han and A. Steckl, ACS Appl. Mater. Interfaces, 2013, 5, 8241.

16 S. Jiang, G. Duan, E. Zussman, A. Greiner and S. Agarwal, ACS Appl. Mater. Interfaces, 2014, 6, 5918.

17 S. Labbaf, H. Ghanbar, E. Stride and M. Edirisinghe, Macromol. Rapid Commun., 2014, 35, 618.

18 W. Liu, C. Ni, D. B. Chase and J. F. Rabolt, ACS Macro Lett., 2013, $2,466$.

19 P. Gupta and G. L. Wilkes, Polymer, 2003, 44, 6353.

20 Q. Ma, W. Yu, X. Dong, J. Wang and G. Liu, Nanoscale, 2014, 6, 2945.

21 F. Bei, X. Dong, J. Wang and G. Liu, RSC Adv., 2015, 5, 12571.

22 Q. Ma, J. Wang, X. Dong, W. Yu and G. Liu, Chem. Eng. J., 2015, 260, 222.

23 Q. Ma, J. Wang, X. Dong, W. Yu and F. Liu, Adv. Funct. Mater., 2015, 25, 2436.

24 X. Xi, Q. Ma, X. Dong, J. Wang, W. Yu and G. Liu, IEEE Trans. Nanotechnol., 2014, 14, 243.

25 K. H. Roh, D. C. Martin and J. Lahann, Nat. Mater., 2005, 4, 759.

26 T. Lin, H. Wang and X. S. Wang, Adv. Mater., 2005, 17, 2699. 
27 Z. Liu, D. D. Sun, P. Guo and J. O. Leckie, Nano Lett., 2007, 7, 1081. 28 S. Chen, H. Hou, P. Hu, J. H. Wendorff, A. Greiner and S. Agarwal, Macromol. Mater. Eng., 2009, 294, 781.

29 J. D. Starr and J. S. Andrew, Chem. Commun., 2013, 49, 4151.

30 J. D. Starr, M. A. K. Budi and J. S. Andrew, J. Am. Ceram. Soc., 2014, 98, 12.

31 G. Chen, Y. Xu, D. G. Yu, D. F. Zhang, N. P. Chatterton and K. N. White, Chem. Commun., 2015, 51, 4623.

32 D. G. Yu, C. Yang, M. Jin, G. R. Williams, H. Zou, X. Wang and S. W. A. Bligh, Colloids Surf., B, 2016, 138, 110.

33 Y. Zhao, X. Cao and L. Jiang, J. Am. Chem. Soc., 2007, 129, 764.

34 C. Yang, D. G. Yu, D. Pan, X. K. Liu, X. Wang, S. W. A. Bligh and G. R. Williams, Acta Biomater., 2016, 35, 77.
35 H. F. Wen, C. Yang, D. G. Yu, X. Y. Li and D. F. Zhang, Chem. Eng. J., 2016, 290, 263.

36 G. Z. Yang, J. J. Li, D. G. Yu, M. F. He, J. H. Yang and G. R. Williams, Acta Biomater., 2017, 36, DOI: 10.1016/j.actbio.2017.01.069.

37 I. G. Loscertales, A. Barrero, I. Guerrero, R. Cortijo, M. Marquez and A. M. Canan-Calvo, Science, 2002, 295, 1695.

38 J. H. Yu, S. V. Fridrikh and G. C. Rutledge, Adv. Mater., 2004, $16,1562$.

39 Q. Xiang, Y. M. Ma, D. G. Yu, M. Jin and G. R. Williams, Appl. Surf. Sci., 2013, 284, 889.

40 C. Li, Z. H. Wang and D. G. Yu, Colloids Surf., B, 2014, 114, 404.

41 G. C. Rutledge and S. V. Fridrikh, Adv. Drug Delivery Rev., 2007, 59, 1384. 